\title{
Fractal Oscillations of Chirp Functions and Applications to Second-Order Linear Differential Equations
}

\author{
Mervan Pašic ${ }^{1}$ and Satoshi Tanaka ${ }^{2}$ \\ ${ }^{1}$ Department of Applied Mathematics, Faculty of Science, University of Zagreb, 10000 Zagreb, Croatia \\ ${ }^{2}$ Okayama University of Science, Okayama 700-0005, Japan
}

Correspondence should be addressed to Satoshi Tanaka; tanaka@xmath.ous.ac.jp

Received 7 December 2012; Accepted 8 January 2013

Academic Editor: Norio Yoshida

Copyright (C) 2013 M. Pašić and S. Tanaka. This is an open access article distributed under the Creative Commons Attribution License, which permits unrestricted use, distribution, and reproduction in any medium, provided the original work is properly cited.

We derive some simple sufficient conditions on the amplitude $a(x)$, the phase $\varphi(x)$, and the instantaneous frequency $\omega(x)$ such that the so-called chirp function $y(x)=a(x) S(\varphi(x))$ is fractal oscillatory near a point $x=x_{0}$, where $\varphi^{\prime}(x)=\omega(x)$ and $S=S(t)$ is a periodic function on $\mathbb{R}$. It means that $y(x)$ oscillates near $x=x_{0}$, and its graph $\Gamma(y)$ is a fractal curve in $\mathbb{R}^{2}$ such that its boxcounting dimension equals a prescribed real number $s \in[1,2)$ and the $s$-dimensional upper and lower Minkowski contents of $\Gamma(y)$ are strictly positive and finite. It numerically determines the order of concentration of oscillations of $y(x)$ near $x=x_{0}$. Next, we give some applications of the main results to the fractal oscillations of solutions of linear differential equations which are generated by the chirp functions taken as the fundamental system of all solutions.

\section{Introduction}

The brilliant heuristic approach of Tricot [1] to the fractal curves such as the graph of functions $y(x)=x^{\alpha} \sin x^{-\beta}$ and $y(x)=x^{\alpha} \cos x^{-\beta}$ gave the main motivation for studying the fractal properties near $x=0$ of graph of oscillatory solutions of various types of differential equations: linear Euler-type equation $y^{\prime \prime}+\lambda x^{-\sigma} y=0$ (see [2]), general second-order linear equation $y^{\prime \prime}+f(x) y=0$ (see [3]) where $f(x)$ satisfies the Hartman-Wintner asymptotic condition near $x=0$, halflinear equation $\left(\left|y^{\prime}\right|^{p-2} y^{\prime}\right)^{\prime}+f(x)|y|^{p-2} y=0$ (see [4]), linear self-adjoint equation $\left(p(x) y^{\prime}\right)^{\prime}+q(x) y=0$ (see [5]), and $p$ Laplace differential equations in an annular domain (see [6]).

A function $y(x)$ is said to be a chirp function if it possesses the form $y(x)=a(x) S(\varphi(x))$, where $a(x)$ and $\varphi(x)$ denote, respectively, the amplitude and phase of $y(x)$, and $S(t)$ is a periodic function on $\mathbb{R}$. In all previously mentioned papers [2-5], authors are dealing with the fractal oscillations of second-order differential equations and are deriving some sufficient conditions on the coefficients of considered equations such that all their solutions $y(x)$ together with the first derivative $y^{\prime}(x)$ admit asymptotic behaviour near $x=0$. It is formally written in the form of a chirp function, that is, $y(x)=a(x) \sin (\varphi(x))$ and $y^{\prime}(x)=b(x) \cos (\varphi(x))$ near $x=$ 0 . According to it, one can say that the asymptotic formula for solutions of considered equations satisfies the chirp-like behaviour near $x=0$ (on the asymptotic formula for solutions near $x=\infty$, see $[7,8])$. Then, in the dependence of a prescribed real number $s \in[1,2)$, authors give some asymptotic conditions on $a(x), b(x)$, and $\varphi(x)$ such that all solutions $y(x)$ are fractal oscillatory near $x=0$ with the fractal dimension $s$.

In this paper, independently of the asymptotic theory of differential equations, we firstly study the fractal oscillations of a chirp function; see Theorems 8 and 11. Second, taking two linearly independent chirp functions $y_{1}(x)$ and $y_{2}(x)$, we generate some new classes of fractal oscillatory linear differential equations which are not considered in [2-5] and have the general solution in the form of $y(x)=c_{1} y_{1}(x)+$ $c_{2} y_{2}(x)$; see Theorems 16 and 17 (on some detailed description of the solution space of the second-order linear differential equations and on their constructions, we refer the reader to $[9,10])$. Finally, we suggest that the reader considers the fractal oscillations near an arbitrary real point $x=x_{0}$ instead of $x=0$ and studies the fractal oscillations near $x=x_{0}$ 
from the left side and from both sides; see Theorem 24. Many examples are considered to show the originality of obtained results.

The chirp functions are also appearing in the timefrequency analysis; see for instance, [11-15] as well as in several applications of the time-frequency analysis; see for instance, [16-20].

\section{Statement of the Main Results}

We study some local asymptotic behaviours of fractal types for the so-called chirp function as follows:

$$
y(x)=a(x) S(\varphi(x)), \quad x \in\left(0, t_{0}\right]
$$

where $t_{0}>0$, the amplitude $a=a(x)$ is a nontrivial function on $\left(0, t_{0}\right]$, the phase $\varphi=\varphi(x)$ is singular at $x=0$ and $S=S(t)$ is a periodic function on $\mathbb{R}$ such as $\cos (t)$ and $\sin (t)$.

The chirp function (1) is sometimes written in the following form in which the so-called instantaneous frequency $\omega(x)$ appears instead of phase $\varphi(x)$ :

$$
y(x)=a(x) S\left(\int_{x}^{t_{0}} \omega(\xi) d \xi\right), \quad x \in\left(0, t_{0}\right]
$$

Let for some $t_{0}>0$, the functions $a \in C\left(\left(0, t_{0}\right]\right), \varphi \in$ $C^{2}\left(\left(0, t_{0}\right]\right)$, and $S \in C^{1}(\mathbb{R})$ satisfy the following basic structural conditions:

$$
\begin{gathered}
a(x)>0, \quad a(x) \text { is bounded on }\left(0, t_{0}\right], \\
\lim _{x \rightarrow+0} \varphi(x)=\infty, \quad \varphi(x)>0, \quad \varphi^{\prime}(x)<0, \quad x \in\left(0, t_{0}\right],
\end{gathered}
$$

for some $\tau>0, \quad|S(t+\tau)|=|S(t)| \quad \forall t \in \mathbb{R}$,

for some $\tau_{0} \in \mathbb{R}, \quad S\left(\tau_{0}\right)=0, \quad S(t) \neq 0$

$$
\forall t \in\left(\tau_{0}, \tau_{0}+\tau\right)
$$

Definition 1. A function $y \in C\left(\left(0, t_{0}\right]\right)$ is oscillatory near $x=0$, if there is a decreasing sequence $x_{n} \in\left(0, t_{0}\right]$ such that $y\left(x_{n}\right)=0$ for $n \in \mathbb{N}$, and $x_{n} \searrow 0$ as $n \rightarrow \infty$; see Figure 1 .

It is easy to prove the next proposition.

Proposition 2. Let $a \in C\left(\left(0, t_{0}\right]\right)$ satisfy $a(x)>0$ on $\left(0, t_{0}\right]$, let $\varphi \in C\left(\left(0, t_{0}\right]\right)$ be strictly decreasing on $\left(0, t_{0}\right]$, and let $S \in C(\mathbb{R})$ satisfy (5). Then, the following two conditions are equivalent:

(i) the chirp function $y(x)=a(x) S(\varphi(x))$ is bounded and oscillatory near $x=0$;

(ii) the amplitude $a(x)$ is bounded and $\lim _{x \rightarrow+0} \varphi(x)=\infty$.

On the qualitative and oscillatory behaviours of solutions of differential equations of several types, we refer the reader to $[7,8]$.

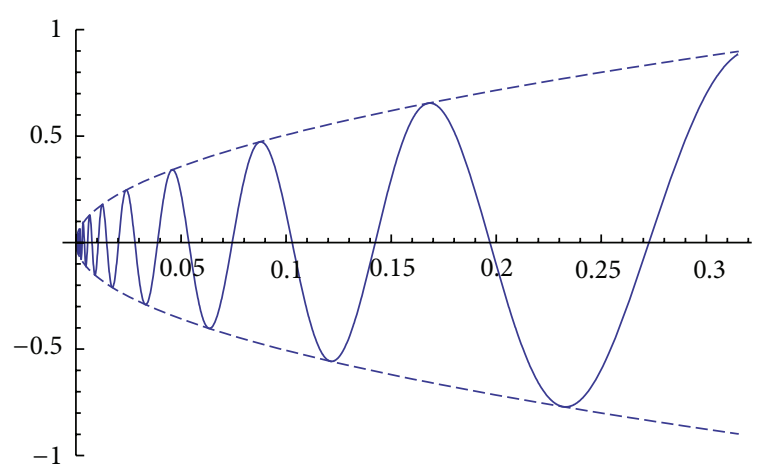

FIGURE 1: $y$ is continuous, bounded, and oscillatory near $x=0$.

Example 3. The following three main types of chirp functions satisfy the conditions (3), (4), and (5):

(i) the so-called $(\alpha, \beta)$-chirps

$$
y_{1}(x)=x^{\alpha} \cos \left(x^{-\beta}\right), \quad y_{2}(x)=x^{\alpha} \sin \left(x^{-\beta}\right),
$$

where $\alpha \geq 0$ and $\beta>0$; the first studies on the $(\alpha, \beta)$ chirps appeared in $[1,13,14]$ from different point of views;

(ii) the so-called logarithmic chirps

$$
y_{1}(x)=x^{\gamma} \cos (\rho \log x), \quad y_{2}(x)=x^{\gamma} \sin (\rho \log x),
$$

where $\gamma>0$ and $\rho \in \mathbb{R}$; this type of chirps appears in definition of the Lamperti transform (see [11]) and in the fundamental system of solutions of the famous Euler equation $y^{\prime \prime}+\lambda x^{-2} y=0$ for $\gamma=1 / 2$ and $\rho=$ $\sqrt{\lambda-1 / 4}$ (see $[21])$;

(iii) the chirp function of exponential type

$$
\begin{aligned}
& y_{1}(x)=x e^{-1 /(2 x)} \cos \left(e^{1 / x}\right) \\
& y_{2}(x)=x e^{-1 /(2 x)} \sin \left(e^{1 / x}\right) .
\end{aligned}
$$

Example 4. Let $y_{1}(x)=a(x) \cos (\varphi(x))$ and $y_{2}(x)=$ $a(x) \sin (\varphi(x)), x \in\left(0, t_{0}\right]$, where the amplitude $a$ and phase $\varphi$ satisfy (3) and (4), respectively. Then, $y(x)=c_{1} y_{1}(x)+$ $c_{2} y_{2}(x), c_{1}^{2}+c_{2}^{2}>0$, is also a chirp function which is bounded and oscillatory near $x=0$. Indeed, if $c_{1}=0$ or $c_{2}=0$, then $y(x)=c_{2} y_{2}(x)$ or $y(x)=c_{1} y_{1}(x)$; since $S(t)=\cos t$ and $S(t)=\sin t$ satisfy (5), both cases $y(x)$ are chirp functions; otherwise, we have $y(x)=A a(x) \sin (\varphi(x)+B)$, where $A=$ $\left(c_{1}^{2}+c_{2}^{2}\right)^{1 / 2}$ and $B=\arctan \left(c_{1} / c_{2}\right)$; obviously, the amplitude $A a(x)$ and the phase $\varphi(x)+B$ satisfy the required conditions (3) and (4), respectively.

Next, let

$$
\Gamma(y)=\left\{\left(t_{1}, t_{2}\right) \in \mathbb{R}^{2}: t_{1} \in\left(0, t_{0}\right], t_{2}=y\left(t_{1}\right)\right\}
$$

denotes the graph of a function $y \in C\left(\left(0, t_{0}\right]\right)$. In this paper, we show that the graph $\Gamma(y)$ of chirp function (1) is a fractal 
curve in $\mathbb{R}^{2}$ in respect to the Minkowski-Bouligand dimension $\operatorname{dim}_{M} \Gamma(y)=s$ (box-counting dimension) and the $s$ dimensional upper and lower Minkowski contents $M^{* s}(\Gamma(y))$ and $M_{*}^{s}(\Gamma(y))$ defined by

$$
\begin{aligned}
\operatorname{dim}_{M} \Gamma(y) & =\lim _{\varepsilon \rightarrow+0}\left(2-\frac{\log \left|\Gamma_{\varepsilon}(y)\right|}{\log \varepsilon}\right), \\
M^{* s}(\Gamma(y)) & =\limsup _{\varepsilon \rightarrow+0}(2 \varepsilon)^{s-2}\left|\Gamma_{\varepsilon}(y)\right|, \\
M_{*}^{s}(\Gamma(y)) & =\liminf _{\varepsilon \rightarrow+0}(2 \varepsilon)^{s-2}\left|\Gamma_{\varepsilon}(y)\right| .
\end{aligned}
$$

Here, $\Gamma_{\varepsilon}(y)$ denotes the $\varepsilon$-neighbourhood of graph $\Gamma(y)$ defined by

$$
\Gamma_{\varepsilon}(y)=\left\{\left(t_{1}, t_{2}\right) \in \mathbb{R}^{2}: d\left(\left(t_{1}, t_{2}\right), \Gamma(y)\right) \leq \varepsilon\right\}, \quad \varepsilon>0,
$$

and $d\left(\left(t_{1}, t_{2}\right), \Gamma(y)\right)$ denotes the distance from $\left(t_{1}, t_{2}\right)$ to $\Gamma(y)$, and $\left|\Gamma_{\varepsilon}(y)\right|$ denotes the Lebesgue measure of $\Gamma_{\varepsilon}(y)$. On the box-counting dimension and the $s$-dimensional Minkowski content, we refer the reader to [22-27].

The main fractal properties considered in the paper are given in the next definitions.

Definition 5. For a given real number $s \in[1,2)$ and a function $y \in C\left(\left(0, t_{0}\right]\right)$, which is bounded on $\left(0, t_{0}\right]$ and oscillatory near $x=0$, it is said that $y(x)$ is fractal oscillatory near $x=0$ with the fractal dimension $s$, if

$$
\operatorname{dim}_{M} \Gamma(y)=s, \quad 0<M_{*}^{s}(\Gamma(y)) \leq M^{* s}(\Gamma(y))<\infty .
$$

On the contrary, if there is no any $s \in[1,2)$ such that $\Gamma(y)$ satisfies (12), then $y(x)$ is not fractal oscillatory near $x=0$.

Fractal oscillations can be understood also as a refinement of rectifiable and nonrectifiable oscillations. They are recently studied in $[3,4,21,28-30]$.

Example 6. The chirp functions

$$
y(x)=x \cos \left(\frac{1}{x}\right), \quad y(x)=x \sin \left(\frac{1}{x}\right)
$$

are not fractal oscillatory near $x=0$. In fact, $M^{* 1}(\Gamma(y))=\infty$ and $\operatorname{dim}_{M} \Gamma(y)=1$ (see [2]). It also implies that $M^{* s}(\Gamma(y))=$ 0 for all $s \in(1,2)$ (see $[1,23]$ ), and thus the statement (12) is not satisfied for any $s \in[1,2)$. Hence, $y_{1}$ and $y_{2}$ are not fractal oscillatory near $x=0$.

Example 7. Let $\rho>0$. It is clear that the chirp functions

$$
y(x)=\sqrt{x} \cos (\rho \log x), \quad y(x)=\sqrt{x} \sin (\rho \log x)
$$

are oscillatory near $x=0$. Moreover, the length of $\Gamma(y)$ is finite (see [21]), and therefore we observe that $0<$ $M_{*}^{1}(\Gamma(y)) \leq M^{* 1}(\Gamma(y))<\infty$ and $\operatorname{dim}_{M} \Gamma(y)=1$ (see [22]). Thus, such chirp functions are fractal oscillatory near $x=0$ with the fractal dimension 1.
In order to show that the chirp function (1) is fractal oscillatory near $x=0$, we need to impose on amplitude $a(x)$ the following additional structural condition:

$$
a \in C^{1}\left(\left(0, t_{0}\right]\right), \quad a^{\prime}(x) \geq 0 \text { for } x \in\left(0, t_{0}\right] .
$$

Now we are able to state the first main result of the paper.

Theorem 8. Let the functions $a(x), \varphi(x)$, and $S(t)$ satisfy the structural conditions (3), (4), (5), (15), and

$$
\varphi^{\prime \prime}(x)>0 \quad \text { on }\left(0, t_{0}\right], \quad \limsup _{x \rightarrow 0}\left(\frac{1}{-\varphi^{\prime}(x)}\right)^{\prime}<\infty .
$$

Let the amplitude $a(x)$ and the phase $\varphi(x)$ satisfy the following asymptotic conditions near $x=0$ :

$$
\begin{gathered}
\liminf _{x \rightarrow 0}\left(\left[-\varphi^{\prime}(x)\right]^{2-s} \int_{0}^{x} a(\xi) d \xi\right)>0, \\
\limsup _{x \rightarrow 0}[x a(x)]^{(s-1) /(2-s)} \int_{x}^{t_{0}} a(\xi)\left[-\varphi^{\prime}(\xi)\right] d \xi<\infty,
\end{gathered}
$$

where $\in(1,2)$ is a given real number. Then, the chirp function (1) is fractal oscillatory near $x=0$ with the fractal dimension $s$.

Remark 9. Let $s \in(1,2)$. Assume that (15) and (17) hold. Then,

$$
\limsup _{x \rightarrow 0}\left(x a(x)\left[-\varphi^{\prime}(x)\right]^{2-s}\right)<\infty
$$

implies

$$
\limsup _{x \rightarrow 0}\left[\frac{1}{-\varphi^{\prime}(x)}\right]^{s-1} \int_{x}^{t_{0}} a(\xi)\left[-\varphi^{\prime}(\xi)\right] d \xi<\infty
$$

and (18). Therefore, condition (18) is better than (19) in Theorem 8 .

The proof of Remark 9 is presented in the Appendix.

Theorem 8 can be rewritten in the term of $\omega(x)$ instead of $\varphi(x)$ as follows.

Corollary 10. Let the functions $a(x), \omega(x)$, and $S(t)$ satisfy the structural conditions (3), (5), (15), and

$$
\begin{gathered}
\omega \in C^{1}\left(\left(0, t_{0}\right]\right), \quad \omega(x)>0, \quad \omega^{\prime}(x)<0 \\
\text { on }\left(0, t_{0}\right], \omega \notin L^{1}\left(\left(0, t_{0}\right]\right), \\
\limsup _{x \rightarrow 0}\left(\frac{1}{\omega(x)}\right)^{\prime}<\infty .
\end{gathered}
$$

Let the amplitude $a(x)$ and the instantaneous frequency $\omega(x)$ satisfy the following asymptotic conditions near $x=0$ :

$$
\begin{gathered}
\liminf _{x \rightarrow 0}\left([\omega(x)]^{2-s} \int_{0}^{x} a(\xi) d \xi\right)>0, \\
\limsup _{x \rightarrow 0}[x a(x)]^{(s-1) /(2-s)} \int_{x}^{t_{0}} a(\xi) \omega(\xi) d \xi<\infty,
\end{gathered}
$$

where $\in(1,2)$ is a given real number. Then, the chirp function (2) is fractal oscillatory near $x=0$ with the fractal dimension $s$. 
Now, we state analogous result to Theorem 8 in the case of $s=1$, which will be proved in Section 3 .

Theorem 11. Assume that the function $S(t)$ satisfies (5), $a \in$ $C\left(\left[0, t_{0}\right]\right) \cap C^{1}\left(\left(0, t_{0}\right]\right), \varphi \in C^{2}\left(\left(0, t_{0}\right]\right)$ and $\lim _{x \rightarrow+0} \varphi(x)=\infty$. If $a^{\prime} \in L^{1}\left(\left(0, t_{0}\right]\right)$, and $\varphi^{\prime} a \in L^{1}\left(\left(0, t_{0}\right]\right)$, then the chirp function (1) is fractal oscillatory near $x=0$ with the fractal dimension 1 .

In respect to some existing results on the fractal dimension of graph of chirp functions, previous theorems are the most simple and general. It is because in [31, 32] authors require some extra conditions on the chirp function $y(x)$ which are not easy to be satisfied in the application, for instance, the rapid convex-concave properties of $y(x)$ as in [31] and a condition on the curvature of $y(x)$ as in [32].

According to previous theorems, we can show the fractal oscillations of the so-called $(\alpha, \beta)$-chirp as well as logarithmic chirp functions.

Example 12. We consider the $(\alpha, \beta)$-chirp $y(x)=x^{\alpha} S\left(x^{-\beta}\right)$, where $S(t)=\cos t$ or $S(t)=\sin t$ and $\beta>\alpha \geq 0$. It is fractal oscillatory near $x=0$ with the fractal dimension $s=2-$ $(1+\alpha) /(1+\beta)$. In fact, it is easy to see that $a(x)=x^{\alpha}, S(t)$, and $\varphi(x)=x^{-\beta}$ satisfy (3), (4), (5), (15), and (16). When $s=$ $2-(1+\alpha) /(1+\beta)$, we see that

$$
\begin{gathered}
{\left[-\varphi^{\prime}(x)\right]^{2-s} \int_{0}^{x} a(\xi) d \xi=\frac{\beta^{(1+\alpha) /(1+\beta)}}{1+\alpha}>0} \\
{[x a(x)]^{(s-1) /(2-s)} \int_{x}^{t_{0}} a(\xi)\left[-\varphi^{\prime}(\xi)\right] d \xi} \\
=\frac{\beta}{\beta-\alpha}\left(1-x^{\beta-\alpha} t_{0}^{\alpha-\beta}\right) \leq \frac{\beta}{\beta-\alpha} .
\end{gathered}
$$

From Theorem 8 , it follows that $y(x)=x^{\alpha} S\left(x^{-\beta}\right), \beta>\alpha \geq 0$, is fractal oscillatory near $x=0$ with the fractal dimension $s=2-(1+\alpha) /(1+\beta)$.

Example 13. We consider the $(\alpha, \beta)$-chirp $y(x)=x^{\alpha} S\left(x^{-\beta}\right)$ again, where $S(t)=\cos t$ or $S(t)=\sin t$. Now, we assume that $0<\beta<\alpha$. Applying Theorem 8 , we easily see that it is fractal oscillatory near $x=0$ with the fractal dimension 1 .

Example 14. We consider the logarithmic chirp functions

$$
y_{1}(x)=x^{\gamma} \cos (\rho \log x), \quad y_{2}(x)=x^{\gamma} \sin (\rho \log x),
$$

where $\gamma>0$ and $\rho>0$. Put $S(t)=\cos (-t)$ or $S(t)=\sin (-t)$, $a(x)=x^{\gamma}$, and $\varphi(x)=-\rho \log x$. Then, we easily see that $S(t)$ satisfies (5) and that $a^{\prime}(x)=\gamma x^{\gamma-1} \in L^{1}\left(\left(0, t_{0}\right]\right)$ and $a(x) \varphi^{\prime}(x)=-\rho x^{\gamma-1} \in L^{1}\left(\left(0, t_{0}\right]\right)$. Theorem 11 implies that $y_{1}(x)$ and $y_{2}(x)$ are fractal oscillatory near $x=0$ with the fractal dimension 1 .

Question 1. Is it possible to apply Theorem 8 on the exponential chirp given in Example 3(iii)?
Example 15. Let $y_{1}(x)=a(x) \cos (\varphi(x))$ and $y_{2}(x)=$ $a(x) \sin (\varphi(x)), x \in\left(0, t_{0}\right]$, where the amplitude $a(x)$ and the phase $\varphi(x)$ satisfy all assumptions of Theorem 8 for an arbitrary given $s \in(1,2)$. Then, the chirp function $y(x)=$ $c_{1} y_{1}(x)+c_{2} y_{2}(x), c_{1}^{2}+c_{2}^{2}>0$, is also fractal oscillatory near $x=0$ with the fractal dimension $s$. Indeed, similarly as in Example 4, $y(x)$ can be rewritten in the form $y(x)=$ $d_{1} \sin \left(\varphi(x)+d_{2}\right)$, where $d_{1} \neq 0$ and $d_{2} \in \mathbb{R}$. It is clear that the function $S(t)=d_{1} \sin \left(t+d_{2}\right)$ satisfies the required condition (5) and hence Theorem 8 proves that $y(x)$ is fractal oscillatory near $x=0$ with the fractal dimension $s$.

Next, we pay attention to the fractal oscillations of solutions of linear differential equations generated by the system of functions as follows:

$$
y(x)=c_{1} y_{1}(x)+c_{2} y_{2}(x), \quad c_{1}^{2}+c_{2}^{2}>0,
$$

where

$$
\begin{array}{r}
y_{1}(x)=a(x) \cos (\varphi(x)), y_{2}(x)=a(x) \sin (\varphi(x)) \\
x \in\left(0, t_{0}\right]
\end{array}
$$

It is not difficult to check that (25) is the fundamental system of all solutions of the following linear differential equation:

$$
\begin{array}{r}
y^{\prime \prime}+\left(-2 R_{a}-R_{\varphi^{\prime}}\right) y^{\prime}+\left(-R_{a}^{\prime}+R_{a}^{2}+R_{a} R_{\varphi^{\prime}}+\left(\varphi^{\prime}\right)^{2}\right) y=0, \\
x \in\left(0, t_{0}\right],
\end{array}
$$

where $R_{f}=R_{f}(x)=f^{\prime}(x) / f(x)$ for some $C^{1}$-function $f=$ $f(x)$.

Theorem 16. Let the functions $a, \varphi \in C^{2}\left(\left(0, t_{0}\right]\right)$ satisfy structural conditions (3), (4), and (15) as well as the conditions (16), (17), and (18) in respect to a given real number $s \in(1,2)$. Then, every nontrivial solution $y \in C^{2}\left(\left(0, t_{0}\right]\right)$ of $(26)$ is fractal oscillatory near $x=0$ with the fractal dimension s.

With the help of Theorem 11, we can state analogous result to Theorem 16 in the case of $s=1$.

Theorem 17. Assume that $a \in C\left(\left[0, t_{0}\right]\right) \cap C^{2}\left(\left(0, t_{0}\right]\right), a(x) \neq 0$ for $x \in\left(0, t_{0}\right], \varphi \in C^{2}\left(\left(0, t_{0}\right]\right), \lim _{x \rightarrow+0} \varphi(x)=\infty, a^{\prime} \in$ $L^{1}\left(\left(0, t_{0}\right]\right)$, and $\varphi^{\prime} a \in L^{1}\left(\left(0, t_{0}\right]\right)$. Then, every nontrivial solution $y \in C^{2}\left(\left(0, t_{0}\right]\right)$ of $(26)$ is fractal oscillatory near $x=0$ with the fractal dimension 1 .

The previous two theorems will be proved in Section 4 . Assumptions on the coefficients of (26) in general are different to those considered in $[2-5]$.

When $a^{\prime} / a=-(1 / 2) \varphi^{\prime \prime} / \varphi^{\prime}$, then (26) becomes the undamped equation

$$
y^{\prime \prime}+\left(\frac{1}{2} S_{\mathrm{der}}\left(\varphi^{\prime}\right)+\left(\varphi^{\prime}\right)^{2}\right) y=0, \quad x \in\left(0, t_{0}\right]
$$


where $S_{\text {der }}(f)$ denotes the Schwarzian derivative of $f$ defined by

$$
S_{\mathrm{der}}(f)=\frac{f^{\prime \prime}}{f}-\frac{3}{2}\left(\frac{f^{\prime}}{f}\right)^{2} .
$$

Hence, from Theorem 16, we obtain the following consequence.

Corollary 18. Let the functions $a \in C^{2}\left(\left(0, t_{0}\right]\right)$ and $\varphi \in$ $C^{3}\left(\left(0, t_{0}\right]\right)$ satisfy structural conditions (3), (4), and (15) as well as the conditions (16), (17), and (18) in respect to a given real number $s \in(1,2)$. Let $a^{\prime} / a=-(1 / 2) \varphi^{\prime \prime} / \varphi^{\prime}$. Then, every nontrivial solution $y \in C^{2}\left(\left(0, t_{0}\right]\right)$ of $(27)$ is fractal oscillatory near $x=0$ with the fractal dimension $s$.

As a consequence of Theorem 16, and Corollary 18 we derive the following examples for linear differential equations of second order having all the solutions to be fractal oscillatory near $x=0$.

Example 19. The so-called damped chirp equation

$$
\begin{array}{r}
y^{\prime \prime}+\frac{\beta-2 \alpha+1}{x} y^{\prime}+\left(\frac{\beta^{2}}{x^{2 \beta+2}}-\frac{\alpha(\beta-\alpha)}{x^{2}}\right) y=0, \\
x \in\left(0, t_{0}\right],
\end{array}
$$

is fractal oscillatory near $x=0$ with the fractal dimension $2-(1+\alpha) /(1+\beta)$, where $\beta>\alpha \geq 0$. When $a(x)=x^{\alpha}$ and $\varphi(x)=x^{-\beta},(26)$ becomes (29). It is easy to see that (3), (4), (15), and (16) are satisfied. In the same as in Example 12, we see that (17) and (18) hold for $s=2-(1+\alpha) /(1+\beta)$. Hence, Theorem 16 proves that every nontrivial solution of (29) is fractal oscillatory near $x=0$ with the fractal dimension $2-$ $(1+\alpha) /(1+\beta)$.

Now we assume that $0<\beta<\alpha$. Then, Theorem 17 implies that (29) is fractal oscillatory near $x=0$ with the fractal dimension 1 .

Example 20. The following equation

$$
y^{\prime \prime}+\frac{1-2 \gamma}{x} y^{\prime}+\frac{\gamma^{2}+\rho^{2}}{x^{2}} y=0, \quad x \in\left(0, t_{0}\right]
$$

is fractal oscillatory near $x=0$ with the fractal dimension 1 , where $\gamma>0$ and $\rho>0$. In the case where $a(x)=x^{\gamma}$ and $\varphi(x)=-\rho \log x$, (26) becomes (30). We see that $a \in$ $C\left(\left[0, t_{0}\right]\right) \cap C^{2}\left(\left(0, t_{0}\right]\right), \varphi \in C^{2}\left(\left(0, t_{0}\right]\right), \lim _{x \rightarrow+0} \varphi(x)=\infty$, $a^{\prime} \in L^{1}\left(\left(0, t_{0}\right]\right)$, and $\varphi^{\prime} a \in L^{1}\left(\left(0, t_{0}\right]\right)$. Therefore Theorem 17 implies that every nontrivial solution of (30) is fractal oscillatory near $x=0$ with the fractal dimension 1 .

Question 2. What can we say about the application of Theorem 16 on the case of $\varphi(x)$ given in Example 3(iii)?

At the end of this section, we suggest that the reader studies some invariant properties of fractal oscillations of the chirp function (1) in respect to the translation and reflexion. Analogously to Definitions 1 and 5, one can define the fractal oscillations near an arbitrary real point $x=x_{0}$ as follows.

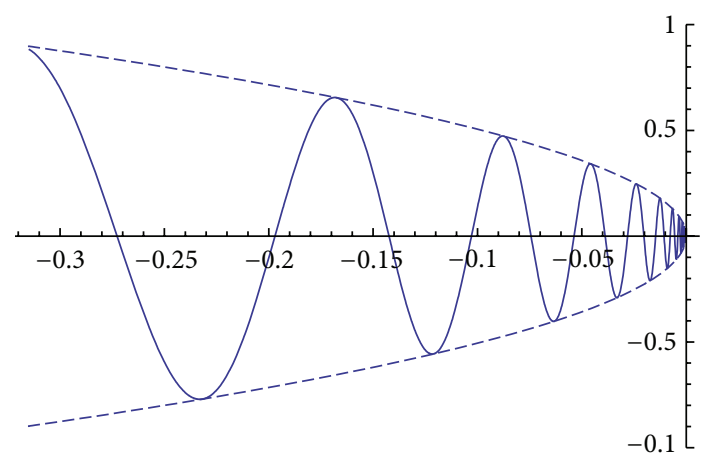

Figure 2: $y$ is oscillatory near $x=0$ from the left side.

Definition 21. Let $x_{0} \in \mathbb{R}$ and $\delta>0$. A function $y \in$ $C\left(\left(x_{0}, x_{0}+\delta\right]\right)$ is oscillatory near $x=x_{0}$, if there is a decreasing sequence $b_{n} \in\left(x_{0}, x_{0}+\delta\right]$ such that $y\left(b_{n}\right)=0$, for $n \in \mathbb{N}$, and $b_{n} \searrow x_{0}$ as $n \rightarrow \infty$. Moreover, if the graph $\Gamma(y)$ satisfies the condition (12) for some $s \in[1,2)$, then $y(x)$ is said to be fractal oscillatory near $x=x_{0}$ with the fractal dimension $s$.

Definition 22. Let $x_{0} \in \mathbb{R}$ and $\delta>0$. It is said that a function $y \in C\left(\left[x_{0}-\delta, x_{0}\right)\right)$ is fractal oscillatory near $x=0$ from the left side with the fractal dimension $s \in[1,2)$, if there is an increasing sequence $a_{n} \in\left[x_{0}-\delta, x_{0}\right)$ such that $y\left(a_{n}\right)=0$ for $n \in \mathbb{N}, a_{n} \nearrow x_{0}$ as $n \rightarrow \infty$, and the graph $\Gamma(y)$ satisfies the condition (12), see Figure 2.

Definition 23. Let $x_{0} \in \mathbb{R}$, and let $\delta>0$. It is said that a function $y \in C\left(\left[x_{0}-\delta, x_{0}\right) \cup\left(x_{0}, x_{0}+\delta\right]\right)$ is two-sided fractal oscillatory near $x=0$ with the fractal dimension $s \in[1,2)$, if there is an increasing sequence $a_{n} \in\left[x_{0}-\delta, x_{0}\right)$ and a decreasing sequence $b_{n} \in\left(x_{0}, x_{0}+\delta\right]$ such that $y\left(a_{n}\right)=$ $y\left(b_{n}\right)=0$ for $n \in \mathbb{N}, a_{n} \nearrow x_{0}$ and $b_{n} \searrow x_{0}$ as $n \rightarrow \infty$, and the graph $\Gamma(y)$ satisfies the condition (12); see Figure 3.

With the help of Theorem 8, we state the following result.

Theorem 24. Let $x_{0} \in \mathbb{R}$, and let $\delta>0$. Let the functions $a, \omega \in C^{1}((0, \delta])$ satisfy structural conditions (3), (4), (5), (15), and (16) as well as conditions (17) and (18) in respect to a given real number $s \in(1,2)$. Then, one has

(i) the chirp function $y(x)=a\left(x-x_{0}\right) S\left(\varphi\left(x-x_{0}\right)\right), x \in$ $\left(x_{0}, x_{0}+\delta\right]$ is fractal oscillatory near $x=x_{0}$ with the fractal dimension s;

(ii) the chirp function $y(x)=a\left(x_{0}-x\right) S\left(\varphi\left(x_{0}-x\right)\right), x \in$ $\left[x_{0}-\delta, x_{0}\right)$ is fractal oscillatory near $x=x_{0}$ from the left side with the fractal dimension s;

(iii) the chirp function $y(x)=a\left(\left|x-x_{0}\right|\right) S\left(\varphi\left(\left|x-x_{0}\right|\right)\right), x \in$ $\left[x_{0}-\delta, x_{0}\right) \cup\left(x_{0}, x_{0}+\delta\right]$ is two-sided fractal oscillatory near $x=x_{0}$ with the fractal dimension $s$. 


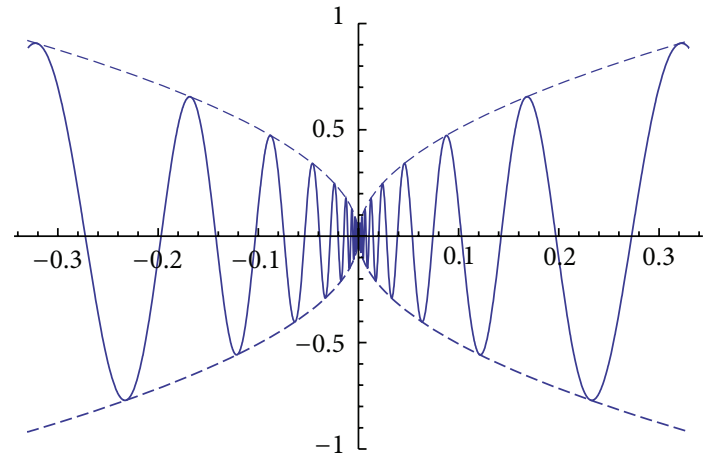

FIGURE 3: $y$ is two-sided oscillatory near $x=0$.

\section{Proof for the Fractal Oscillations of Chirp Functions}

In this section, we give the proofs of the main results dealing with the fractal oscillations of chirp functions.

By Definition 5, it follows that if for a prescribed real number $s \in(1,2)$ there are two positive constants $c_{1}$ and $c_{2}$ such that

$$
c_{1} \varepsilon^{2-s} \leq\left|\Gamma_{\varepsilon}(y)\right| \leq c_{2} \varepsilon^{2-s}, \quad \varepsilon \in\left(0, \varepsilon_{0}\right),
$$

for some $\varepsilon_{0}>0$, then a function $y \in C\left(\left(0, t_{0}\right]\right)$ is fractal oscillatory near $x=0$ with the fractal dimension $s$. The following lemma plays the essential role in the proof of (31).

Lemma 25. Let $y \in C\left(\left(0, t_{0}\right]\right)$ be a bounded function on $\left(0, t_{0}\right]$, and let $a_{n} \in\left(0, t_{0}\right]$ be a decreasing sequence of consecutive zeros of $y(x)$ such that $a_{n} \rightarrow 0$. Let $\varepsilon_{0}>0$, and let $k=k(\varepsilon)$ be an index function satisfying

$$
\left|a_{n}-a_{n+1}\right| \leq \varepsilon \quad \forall n \geq k(\varepsilon), \varepsilon \in\left(0, \varepsilon_{0}\right) .
$$

Then

$$
\begin{array}{r}
\left|\Gamma_{\varepsilon}(y)\right| \geq \sum_{n \geq k(\varepsilon)} \max _{x \in\left[a_{n+1}, a_{n}\right]}|y(x)|\left(a_{n}-a_{n+1}\right) \\
\forall \varepsilon \in\left(0, \varepsilon_{0}\right) .
\end{array}
$$

Proof. It is exactly the same as [3, pp. 2350].

Let us remark that for some $\varepsilon_{0}>0$, we say that a function $k=k(\varepsilon)$ is an index function on $\left(0, \varepsilon_{0}\right)$ if $k:\left(0, \varepsilon_{0}\right) \rightarrow \mathbb{N}$ and $\lim _{\varepsilon \rightarrow 0} k(\varepsilon)=\infty$.

Lemma 26. Let $y \in C[a, b]$. Then,

$$
\left|\Gamma_{\varepsilon}(y)\right| \leq 4 \pi \varepsilon \text { length }(y)+4 \pi \varepsilon^{2}, \quad \text { for every } \varepsilon>0 .
$$

Proof. Let $\varepsilon>0$. Set $x_{1}=a$, and set

$$
\begin{gathered}
x_{i+1}=\max \left\{x \in\left[x_{i}, b\right]: d\left((t, y(t)),\left(x_{i}, y\left(x_{i}\right)\right)\right)\right. \\
\left.\leq \varepsilon \text { for } t \in\left[x_{i}, x\right]\right\},
\end{gathered}
$$

for $i=1,2, \ldots$. Then, there exists $n \geq 2$ such that $x_{n}=b$. Set $N=\max \left\{n \in \mathbf{N}: x_{n}<b\right\}$. We see that $N \geq 1$,

$$
a=x_{1}<x_{2}<\cdots<x_{i}<x_{i+1}<\cdots<x_{N}<x_{N+1}=b,
$$

and if $N \geq 2$, then

$$
d\left(\left(x_{i}, y\left(x_{i}\right)\right),\left(x_{i+1}, y\left(x_{i+1}\right)\right)\right)=\varepsilon, \quad i=1,2, \ldots, N-1 .
$$

We will show that

$$
\Gamma_{\varepsilon}(y) \subset \bigcup_{i=1}^{N} B_{2 \varepsilon}\left(x_{i}, y\left(x_{i}\right)\right),
$$

where

$$
B_{2 \varepsilon}\left(t_{1}, t_{2}\right)=\left\{\left(\tau_{1}, \tau_{2}\right) \in \mathbf{R}^{2}: d\left(\left(t_{1}, t_{2}\right),\left(\tau_{1}, \tau_{2}\right)\right) \leq 2 \varepsilon\right\} .
$$

Let $\left(\tau_{1}, \tau_{2}\right) \in \Gamma_{\varepsilon}(y)$. Then there exists $\xi \in[a, b]$ such that $d\left(\left(\tau_{1}, \tau_{2}\right),(\xi, y(\xi))\right) \leq \varepsilon$. Because of the definition of $x_{i+1}$, we find that $\xi \in\left[x_{i}, x_{i+1}\right]$ for some $i \in\{1,2, \ldots, N\}$, so that

$$
d\left((\xi, y(\xi)),\left(x_{i}, y\left(x_{i}\right)\right)\right) \leq \varepsilon \text {. }
$$

Hence, it follows that

$$
\begin{aligned}
d\left(\left(\tau_{1}, \tau_{2}\right),\left(x_{i}, y\left(x_{i}\right)\right)\right) \leq & d\left(\left(\tau_{1}, \tau_{2}\right),(\xi, y(\xi))\right) \\
& +d\left((\xi, y(\xi)),\left(x_{i}, y\left(x_{i}\right)\right)\right) \leq 2 \varepsilon,
\end{aligned}
$$

which means that $\left(\tau_{1}, \tau_{2}\right) \in B_{2 \varepsilon}\left(x_{i}, y\left(x_{i}\right)\right)$. Therefore, we obtain (38). By (38), we conclude that

$$
\left|\Gamma_{\varepsilon}(y)\right| \leq \sum_{i=1}^{N}\left|B_{2 \varepsilon}\left(x_{i}, y\left(x_{i}\right)\right)\right|=4 N \pi \varepsilon^{2} .
$$

When $N=1$, from (42), it follows that

$$
\left|\Gamma_{\varepsilon}(y)\right| \leq 4 \pi \varepsilon^{2} \leq 4 \pi \varepsilon \text { length }(y)+4 \pi \varepsilon^{2} .
$$

Now, we assume that $N \geq 2$. We observe that

$$
\begin{aligned}
\text { length }(y) & =\sum_{i=1}^{N} \text { length }\left(\left.y\right|_{\left[x_{i}, x_{i+1}\right]}\right) \\
& \geq \sum_{i=1}^{N} d\left(\left(x_{i}, y\left(x_{i}\right)\right),\left(x_{i+1}, y\left(x_{i+1}\right)\right)\right) \\
& \geq \sum_{i=1}^{N-1} d\left(\left(x_{i}, y\left(x_{i}\right)\right),\left(x_{i+1}, y\left(x_{i+1}\right)\right)\right) \\
& =(N-1) \varepsilon,
\end{aligned}
$$

that is,

$$
N \varepsilon \leq \text { length }(y)+\varepsilon .
$$

Combining (42) with (45), we obtain

$$
\left|\Gamma_{\varepsilon}(y)\right| \leq 4 \pi \varepsilon \text { length }(y)+4 \pi \varepsilon^{2} .
$$


Lemma 27. Let $y \in C\left(0, t_{0}\right]$ be bounded on $\left(0, t_{0}\right]$, and let $\varepsilon>0$. Then, there exists $C>0$ such that, for every $\varepsilon>0$ and $t \in\left(0, t_{0}\right]$,

$$
\left|\Gamma_{\varepsilon}(y)\right| \leq C\left[t \sup _{x \in(0, t]}|y(x)|+\varepsilon \operatorname{length}\left(\left.y\right|_{\left[t, t_{0}\right]}\right)+\varepsilon+\varepsilon^{2}\right] .
$$

Proof. Let $\varepsilon>0$, and let $t \in\left(0, t_{0}\right]$. From the geometry point of view, it is clear that

$$
\left|\Gamma_{\varepsilon}\left(\left.y\right|_{(0, t]}\right)\right| \subset R,
$$

where $R$ is a rectangle in $\mathbf{R}^{2}$ defined by

$$
\begin{aligned}
R=\{ & \left(t_{1}, t_{2}\right): t_{1} \in[-\mathcal{\varepsilon}, t+\varepsilon], \\
& \left.t_{2} \in\left[-\varepsilon-\sup _{x \in(0, t]}|y(x)|, \varepsilon+\sup _{x \in(0, t]}|y(x)|\right]\right\} .
\end{aligned}
$$

Hence, we have

$$
\begin{aligned}
\left|\Gamma_{\varepsilon}\left(y_{(0, t]}\right)\right| \leq & (2 \varepsilon+t)\left(2 \varepsilon+2 \sup _{x \in(0, t]}|y(x)|\right) \\
= & 4 \varepsilon^{2}+2\left(t+2 \sup _{x \in(0, t]}|y(x)|\right) \varepsilon \\
& +2 t \sup _{x \in(0, t]}|y(x)| \\
\leq & 4 \varepsilon^{2}+C_{1} \varepsilon+2 t \sup _{x \in(0, t]}|y(x)|,
\end{aligned}
$$

where $C_{1}=2\left(t_{0}+2 \sup _{x \in\left(0, t_{0}\right]}|y(x)|\right)$. Lemma 26 implies that

$$
\left|\Gamma_{\varepsilon}\left(\left.y\right|_{\left[t, t_{0}\right]}\right)\right| \leq 4 \pi \varepsilon \text { length }\left(\left.y\right|_{\left[t, t_{0}\right]}\right)+4 \pi \varepsilon^{2} .
$$

Consequently, we see that

$$
\begin{aligned}
\left|\Gamma_{\varepsilon}(y)\right| \leq & \left|\Gamma_{\varepsilon}\left(\left.y\right|_{(0, t]}\right)\right|+\left|\Gamma_{\varepsilon}\left(\left.y\right|_{\left[t, t_{0}\right]}\right)\right| \\
\leq & 4 \varepsilon^{2}+C_{1} \varepsilon+2 t \sup _{x \in(0, t]}|y(x)| \\
& +4 \pi \varepsilon \operatorname{length}\left(\left.y\right|_{\left[t, t_{0}\right]}\right)+4 \pi \varepsilon^{2} \\
\leq & C\left[t \sup _{x \in(0, t]}|y(x)|+\varepsilon \text { length }\left(\left.y\right|_{\left[t, t_{0}\right]}\right)+\varepsilon+\varepsilon^{2}\right],
\end{aligned}
$$

where $C=\max \left\{C_{1}, 4(\pi+1)\right\}$.

Lemma 28. Let $y \in C^{1}\left(0, t_{0}\right]$ be bounded on $\left(0, t_{0}\right]$. Assume that

$$
\begin{gathered}
\int_{0}^{t_{0}}\left|y^{\prime}(x)\right| d x=\infty, \\
\limsup _{x \rightarrow 0}\left[x \sup _{\xi \in(0, x]}|y(\xi)|\right]^{(s-1) /(2-s)} \int_{x}^{t_{0}}\left|y^{\prime}(\xi)\right| d \xi<\infty,
\end{gathered}
$$

for some $\in(1,2)$. Then, there exists $c_{2}>0$ such that

$$
\left|\Gamma_{\varepsilon}(y)\right| \leq c_{2} \varepsilon^{2-s}, \quad \varepsilon \in(0,1) .
$$

Proof. Let $\varepsilon \in(0,1)$ and let $s \in(1,2)$ be satisfy (54). By (53), there exists $x_{1} \in\left(0, t_{0}\right)$, which depends on $\varepsilon$ and $s$, such that

$$
\int_{x_{1}}^{t_{0}}\left|y^{\prime}(t)\right| d t=\varepsilon^{1-s}
$$

Therefore, we have

$$
\begin{aligned}
\operatorname{length}\left(\left.y\right|_{\left[x_{1}, t_{0}\right]}\right) & =\int_{x_{1}}^{t_{0}} \sqrt{1+\left|y^{\prime}(x)\right|^{2}} d x \\
& \leq \int_{x_{1}}^{t_{0}}\left(1+\left|y^{\prime}(x)\right|\right) d x \\
& =t_{0}-x_{1}+\varepsilon^{1-s} \\
& \leq t_{0}+\varepsilon^{1-s} .
\end{aligned}
$$

By (54), there exists $C_{1}>0$ such that

$$
\left[x \sup _{\xi \in(0, x]}|y(\xi)|\right]^{(s-1) /(2-s)} \int_{x}^{t_{0}}\left|y^{\prime}(\xi)\right| d \xi \leq C_{1}, \quad x \in\left(0, t_{0}\right],
$$

which implies that

$$
\left[x_{1} \sup _{\xi \in\left(0, x_{1}\right]}|y(\xi)|\right]^{(s-1) /(2-s)} \int_{x_{1}}^{t_{0}}\left|y^{\prime}(\xi)\right| d \xi \leq C_{1}
$$

that is,

$$
x_{1} \sup _{\xi \in\left(0, x_{1}\right]}|y(\xi)| \leq C_{2} \varepsilon^{2-s},
$$

where $C_{2}=C_{1}^{(2-s) /(s-1)}$. From Lemma 27, (57), and (60), it follows that

$$
\begin{aligned}
\left|\Gamma_{\varepsilon}(y)\right| & \leq C\left[C_{2} \varepsilon^{2-s}+\varepsilon\left(t_{0}+\varepsilon^{1-s}\right)+\varepsilon+\varepsilon^{2}\right] \\
& =C\left[\left(C_{2}+1\right)+\left(t_{0}+1\right) \varepsilon^{s-1}+\varepsilon^{s}\right] \varepsilon^{2-s} \\
& \leq C\left(C_{2}+t_{0}+3\right) \varepsilon^{2-s}, \quad \varepsilon \in(0,1) .
\end{aligned}
$$

In order to show Theorems 8 and 11, we need the following two geometric lemmas.

Lemma 29 (see [1]). If $\Gamma \subseteq \mathbb{R}^{2}$ is a simple curve (i.e., its parameterization is a bijection) and length $(\Gamma)<\infty$, then

$$
\text { length }(\Gamma)=\lim _{\varepsilon \rightarrow 0} \frac{\left|\Gamma_{\varepsilon}\right|}{2 \varepsilon},
$$

where $\Gamma_{\varepsilon}$ denotes the $\varepsilon$-neighborhood of the graph $\Gamma$. 
Now, we are able to prove Theorem 8.

Proof of Theorem 8. Let $s \in(1,2)$, and let $y(x)$ be a chirp function given by (1). We note here that it is enough to show that $y(x)$ satisfies (31).

At the first, let $x_{n}$ be a sequence defined by $x_{n}=\varphi^{-1}\left(\tau_{0}+\right.$ $n \tau)$ for all sufficiently large $n \in \mathbb{N}$. From (4), it follows that $\varphi^{-1}(t)$ is decreasing. Hence, $x_{n}$ is decreasing as well as $x_{n} \rightarrow$ 0 as $n \rightarrow \infty$ because of $\lim _{x \rightarrow+0} \varphi(x)=\infty$ (see (4)). We note that $y\left(x_{n}\right)=0$ and $y(x) \neq 0$ on $\left(x_{n+1}, x_{n}\right)$ for all sufficiently large $n \in \mathbb{N}$. Also, $-1 / \varphi^{\prime}(x)$ is an increasing function because of (16). The mean value theorem shows that

$$
\frac{\tau}{-\varphi^{\prime}\left(x_{n+1}\right)} \leq x_{n}-x_{n+1} \leq \frac{\tau}{-\varphi^{\prime}\left(x_{n}\right)} \text {. }
$$

Now, let $\varepsilon_{0} \in(0,1)$. Let $k(\varepsilon)$ be the smallest natural number satisfying

$$
\frac{\tau}{-\varphi^{\prime}\left(x_{k(\varepsilon)}\right)} \leq \varepsilon \quad \forall \varepsilon \in\left(0, \varepsilon_{0}\right) .
$$

Such $k(\varepsilon)$ exists for every $\varepsilon \in\left(0, \varepsilon_{0}\right)$, since $x_{n} \rightarrow 0$ as $n \rightarrow$ $\infty$ and $\lim _{x \rightarrow+0} \varphi^{\prime}(x)=-\infty$ (this equality is true because $\varphi^{\prime} \notin L^{1}\left(0, t_{0}\right)$ since $\left.\lim _{x \rightarrow+0} \varphi(x)=\infty\right)$. Moreover, since $x_{n}$ is decreasing and $-1 / \varphi^{\prime}(x)$ is increasing, we obtain

$$
-\varphi^{\prime}\left(x_{n}\right) \geq \tau \varepsilon^{-1} \quad \forall n \geq k(\varepsilon) .
$$

Combining (63) and (65), it is easy to deduce that such defined $k(\varepsilon)$ satisfies condition (32).

By (16), there exists $L>0$ such that $\left(1 /\left(-\varphi^{\prime}(x)\right)\right)^{\prime} \leq L$ for $x \in\left(0, t_{0}\right]$, which means that

$$
-\frac{-\varphi^{\prime \prime}(x)}{-\varphi^{\prime}(x)} \leq-L \varphi^{\prime}(x), \quad x \in\left(0, t_{0}\right] .
$$

Integrating (66) on $\left[\varphi^{-1}(t+2 \tau), \varphi^{-1}(t)\right]$, we have

$$
\log \frac{-\varphi^{\prime}\left(\varphi^{-1}(t+2 \tau)\right)}{-\varphi^{\prime}\left(\varphi^{-1}(t)\right)} \leq 2 L \tau, \quad t \in\left[\varphi\left(t_{0}\right), \infty\right)
$$

that is,

$$
-\varphi^{\prime}\left(\varphi^{-1}(t)\right) \geq-e^{-2 L \tau} \varphi^{\prime}\left(\varphi^{-1}(t+\tau)\right), \quad t \in\left[\varphi\left(t_{0}\right), \infty\right) .
$$

By the definition of $k(\varepsilon)$ and (64), we see that

$$
\frac{\tau}{-\varphi^{\prime}\left(x_{k(\varepsilon)-1}\right)}>\varepsilon
$$

Hence, from (68), it follows that

$$
\begin{aligned}
\tau \varepsilon^{-1} & >-\varphi^{\prime}\left(x_{k(\varepsilon)-1}\right) \\
& =-\varphi^{\prime}\left(\varphi^{-1}\left(\tau_{0}+(k(\varepsilon)-1) \tau\right)\right) \\
& \geq-e^{-2 L \tau} \varphi^{\prime}\left(\varphi^{-1}\left(\tau_{0}+(k(\varepsilon)-1) \tau+2 \tau\right)\right) \\
& =-e^{-2 L \tau} \varphi^{\prime}\left(\varphi^{-1}\left(\tau_{0}+(k(\varepsilon)+1) \tau\right)\right) \\
& =-e^{-2 L \tau} \varphi^{\prime}\left(x_{k(\varepsilon)+1}\right),
\end{aligned}
$$

which implies that

$$
-\varphi^{\prime}\left(x_{k(\varepsilon)+1}\right) \leq M \varepsilon^{-1}, \quad \varepsilon>0,
$$

where $M=\tau e^{2 L \tau}$.

Next, it is clear that the assumption (5) ensures a real number $M_{0}>0$ such that

$$
M_{0}=\max _{t \in \mathbb{R}}|S(t)|=\max _{t \in[0, \tau]}|S(t)| .
$$

Hence, from (15), we have

$$
\begin{aligned}
\max _{x \in\left[x_{n+1}, x_{n}\right]}|y(x)| & =\max _{x \in\left[x_{n+1}, x_{n}\right]}(a(x)|S(\varphi(x))|) \\
& \geq a\left(x_{n+1}\right) \max _{x \in\left[x_{n+1}, x_{n}\right]}|S(\varphi(x))| \\
& =M_{0} a\left(x_{n+1}\right) .
\end{aligned}
$$

Now, from (63) and (73), it follows that

$$
\begin{aligned}
\max _{x \in\left[x_{n+1}, x_{n}\right]}|y(x)|\left(x_{n}-x_{n+1}\right) & \geq M_{0} a\left(x_{n+1}\right)\left(x_{n}-x_{n+1}\right) \\
& \geq \tau M_{0} \frac{a\left(x_{n+1}\right)}{-\varphi^{\prime}\left(x_{n+1}\right)} \\
& =\tau M_{0} \frac{a\left(\varphi^{-1}\left(\tau_{0}+(n+1) \tau\right)\right)}{-\varphi^{\prime}\left(\varphi^{-1}\left(\tau_{0}+(n+1) \tau\right)\right)} .
\end{aligned}
$$

Since $\varphi^{-1}(t)$ is decreasing as well as $-1 / \varphi^{\prime}(x)$ is increasing and $a(x)$ is nondecreasing and positive, we conclude that the function

$$
\frac{a\left(\varphi^{-1}(t)\right)}{-\varphi^{\prime}\left(\varphi^{-1}(t)\right)} \text { is nonincreasing. }
$$

Hence, we observe that

$$
\begin{aligned}
& \sum_{n \geq k(\varepsilon)} \max _{x \in\left[x_{n+1}, x_{n}\right]}|y(x)|\left(x_{n}-x_{n+1}\right) \\
& \geq \tau M_{0} \sum_{n \geq k(\varepsilon)} \frac{a\left(\varphi^{-1}\left(\tau_{0}+(n+1) \tau\right)\right)}{-\varphi^{\prime}\left(\varphi^{-1}\left(\tau_{0}+(n+1) \tau\right)\right)} \\
& \geq \tau M_{0} \int_{k(\varepsilon)}^{\infty} \frac{a\left(\varphi^{-1}\left(\tau_{0}+(t+1) \tau\right)\right)}{-\varphi^{\prime}\left(\varphi^{-1}\left(\tau_{0}+(t+1) \tau\right)\right)} d t \\
& =M_{0} \int_{0}^{\varphi^{-1}\left(\tau_{0}+(k(\varepsilon)+1) \tau\right)} a(\xi) d \xi \\
& =M_{0} \int_{0}^{x_{k(\varepsilon)+1}} a(\xi) d \xi .
\end{aligned}
$$

Next, from assumption (17), we get some $M_{1}>0$ such that

$$
\int_{0}^{x} a(\xi) d \xi \geq M_{1}\left[-\varphi^{\prime}(x)\right]^{-(2-s)}, \quad x \in\left(0, t_{0}\right] .
$$


Now, from (76), (77), and (71), we obtain

$$
\begin{gathered}
\sum_{n \geq k(\varepsilon)} \max _{x \in\left[x_{n+1}, x_{n}\right]}|y(x)|\left(x_{n}-x_{n+1}\right) \\
\geq M_{0} M_{1}\left[-\varphi^{\prime}\left(x_{k(\varepsilon)+1}\right)\right]^{-(2-s)} \\
\geq M_{0} M_{1}\left(M \varepsilon^{-1}\right)^{-(2-s)} \\
\quad=M_{0} M_{1} M^{-(2-s)} \varepsilon^{2-s} .
\end{gathered}
$$

Thus, using Lemma 25, we have

$$
\left|\Gamma_{\varepsilon}(y)\right| \geq c_{1} \varepsilon^{2-s}, \quad \varepsilon \in\left(0, \varepsilon_{0}\right),
$$

for some $c_{1}>0$.

Next we prove that $y(x)$ satisfies (53). Assume to the contrary that

$$
\int_{0}^{t_{0}}\left|y^{\prime}(x)\right| d x<\infty
$$

Then, we have

$$
\begin{aligned}
\text { length }(y) & =\int_{0}^{t_{0}} \sqrt{1+\left|y^{\prime}(x)\right|^{2}} d x \\
& \leq \int_{0}^{t_{0}}\left(1+\left|y^{\prime}(x)\right|\right) d x<\infty .
\end{aligned}
$$

From Lemma 29, it follows that

$$
\text { length }(y)=\lim _{\varepsilon \rightarrow+0} \frac{\left|\Gamma_{\varepsilon}(y)\right|}{2 \varepsilon} .
$$

On the other hand, by (79), we see that

$$
\lim _{\varepsilon \rightarrow+0} \frac{\left|\Gamma_{\varepsilon}(y)\right|}{2 \varepsilon}=\infty .
$$

This is a contaradiction, and hence $y(x)$ satisfies (53).

Finally, we show that $y(x)$ also satisfies inequality (54). Because of (5), we obtain a constant $c>0$ such that

$$
\begin{gathered}
\max _{t \in \mathbb{R}}|S(t)|=\max _{t \in[0, \tau]}|S(t)| \leq c, \\
\max _{t \in \mathbb{R}}\left|S^{\prime}(t)\right|=\max _{t \in[0, \tau]}\left|S^{\prime}(t)\right| \leq c .
\end{gathered}
$$

By (15), we find that

$$
\sup _{t \in(0, x]}|y(t)|=\sup _{t \in(0, x]}|a(t)||S(\varphi(t))| \leq c a(x) .
$$

Hence, from (1) and (15), it follows that

$$
\left|y^{\prime}(x)\right| \leq c\left(a^{\prime}(x)+a(x)\left[-\varphi^{\prime}(x)\right]\right),
$$

and therefore

$$
\int_{x}^{t_{0}}\left|y^{\prime}(\xi)\right| d \xi \leq c a\left(t_{0}\right)+c \int_{x}^{t_{0}} a(\xi)\left[-\varphi^{\prime}(\xi)\right] d \xi .
$$

Using (85) and (87), we see that

$$
\begin{aligned}
& {\left[x \sup _{\xi \in(0, x]}|y(\xi)|\right]^{(s-1) /(2-s)} \int_{x}^{t_{0}}\left|y^{\prime}(\xi)\right| d \xi} \\
& \leq c a\left(t_{0}\right)[c x a(x)]^{(s-1) /(2-s)} \\
& \quad+c[c x a(x)]^{(s-1) /(2-s)} \int_{x}^{t_{0}} a(\xi)\left[-\varphi^{\prime}(\xi)\right] d \xi \\
& \leq c a\left(t_{0}\right)\left[c t_{0} a\left(t_{0}\right)\right]^{(s-1) /(2-s)} \\
& \quad+c^{1 /(2-s)}[x a(x)]^{(s-1) /(2-s)} \int_{x}^{t_{0}} a(\xi)\left[-\varphi^{\prime}(\xi)\right] d \xi .
\end{aligned}
$$

By (18) we conclude that (54) holds.

By Lemma 28, there exists $c_{2}>0$ such that

$$
\left|\Gamma_{\varepsilon}(y)\right| \leq c_{2} \varepsilon^{2-s}, \quad \varepsilon \in\left(0, \varepsilon_{0}\right) .
$$

Thus, we have proved that the chirp function $y(x)$ given by $(1)$ satisfies the desired inequality (31). This completes the proof of Theorem 8 .

Proof of Theorem 11. Let $y(x)$ be the chirp function (1). It is easy to see that $y(x)$ is oscillatory near $x=0$. By (5), there exists $M>0$ such that

$$
|S(t)| \leq M, \quad\left|S^{\prime}(t)\right| \leq M, \quad t \in \mathbb{R} .
$$

Hence, we observe that

$$
\begin{aligned}
\left|y^{\prime}(x)\right| & \leq\left|a^{\prime}(x) S(\varphi(x))\right|+\left|a(x) S(\varphi(x)) \varphi^{\prime}(x)\right| \\
& \leq M\left|a^{\prime}(x)\right|+M\left|a(x) \varphi^{\prime}(x)\right|,
\end{aligned}
$$

for $x \in\left(0, t_{0}\right]$. From (81), it follows that length $(y)<\infty$. Lemma 29 shows that

$$
\text { length }(y)=\lim _{\varepsilon \rightarrow 0} \frac{\left|\Gamma_{\varepsilon}(y)\right|}{2 \varepsilon},
$$

which implies that $\varepsilon$ length $(\Gamma) \leq\left|\Gamma_{\varepsilon}(y)\right| \leq 4$ clength $(\Gamma), \varepsilon \in$ $\left(0, \varepsilon_{0}\right)$, for some $\varepsilon_{0}>0$. Therefore, $y(x)$ is fractal oscillatory near $x=0$ with the fractal dimension 1 .

\section{Proof for the Fractal Oscillations of (26)}

In this section, we give the proofs for the fractal oscillations of the linear second-order differential equation (26) considered as an application of the main results on the fractal oscillation of chirp functions.

Before we present the proofs of Theorems 16 and 17, we make the following observation. Since

$$
y_{1}(x)=a(x) \cos (\varphi(x)), \quad y_{2}(x)=a(x) \sin (\varphi(x))
$$

are solutions of (26), we see that $y(x)=c_{1} y_{1}(x)+c_{2} y_{2}(x)$ is a fundamental system of all solutions of (26). Assume that $c_{1}^{2}+c_{2}^{2}>0$, and set $S(t)=c_{1} \sin t+c_{2} \cos t$. Then, $S(t)$ clearly satisfies (5). 
Proof of Theorem 16. Applying Theorem 8 on $y(x)=$ $c_{1} y_{1}(x)+c_{2} y_{2}(x)$, we conclude that $y(x)$ is fractal oscillatory with the fractal dimension $s$.

Proof of Theorem 17. Theorem 11 implies that $y(x)=$ $c_{1} y_{1}(x)+c_{2} y_{2}(x)$ is fractal oscillatory near $x=0$ with the fractal dimension 1 .

\section{Appendix}

Proof of Remark 9. By (17), there exists $c_{1}>0$ such that

$$
c_{1} \leq\left[-\varphi^{\prime}(x)\right]^{2-s} \int_{0}^{x} a(\xi) d \xi, \quad x \in\left(0, t_{0}\right] .
$$

From (15), it follows that

$$
\begin{array}{r}
c_{1} \leq\left[-\varphi^{\prime}(x)\right]^{2-s} a(x) \int_{0}^{x} d \xi=x a(x)\left[-\varphi^{\prime}(x)\right]^{2-s}, \\
x \in\left(0, t_{0}\right],
\end{array}
$$

which implies that

$$
\frac{1}{-\varphi^{\prime}(x)} \leq c_{1}^{-1 /(2-s)} x^{1 /(2-s)}[a(x)]^{1 /(2-s)}, \quad x \in\left(0, t_{0}\right] .
$$

By (19), there exists $c_{2}>0$ such that

$$
x a(x)\left[-\varphi^{\prime}(x)\right]^{2-s} \leq c_{2}, \quad x \in\left(0, t_{0}\right]
$$

and hence

$$
-\varphi^{\prime}(x) \leq c_{2}^{1 /(2-s)} x^{-1 /(2-s)}[a(x)]^{-1 /(2-s)}, \quad x \in\left(0, t_{0}\right] .
$$

Therefore, we see that

$$
\begin{aligned}
& {\left[\frac{1}{-\varphi^{\prime}(x)}\right]^{s-1} \int_{x}^{t_{0}} a(\xi)\left[-\varphi^{\prime}(\xi)\right] d \xi} \\
& \leq c_{1}^{-(s-1) /(2-s)} x^{(s-1) /(2-s)}[a(x)]^{(s-1) /(2-s)} c_{2}^{1 /(2-s)} \\
& \quad \times \int_{x}^{t_{0}} \xi^{-1 /(2-s)}[a(\xi)]^{-(s-1) /(2-s)} d \xi \\
& \leq c_{3} x^{(s-1) /(2-s)}[a(x)]^{(s-1) /(2-s)}[a(x)]^{-(s-1) /(2-s)} \\
& \quad \times \int_{x}^{t_{0}} \xi^{-1 /(2-s)} d \xi \\
& =c_{3} \frac{2-s}{s-1}\left(1-x^{(s-1) /(2-s)} t_{0}^{-(s-1) /(2-s)}\right) \\
& \leq c_{3} \frac{2-s}{s-1}, \quad x \in\left(0, t_{0}\right]
\end{aligned}
$$

so that (20) holds. We conclude that

$$
\begin{aligned}
& {[x a(x)]^{(s-1) /(2-s)} \int_{x}^{t_{0}} a(\xi)\left[-\varphi^{\prime}(\xi)\right] d \xi} \\
& =\left(x a(x)\left[-\varphi^{\prime}(x)\right]^{2-s}\right)^{(s-1) /(2-s)} \\
& \quad \times\left(\left[\frac{1}{-\varphi^{\prime}(x)}\right]^{s-1} \int_{x}^{t_{0}} a(\xi)\left[-\varphi^{\prime}(\xi)\right] d \xi\right) .
\end{aligned}
$$

By (19) and (20), we conclude that (18) is satisfied. The proof of Remark 9 is complete.

\section{References}

[1] C. Tricot, Curves and Fractal Dimension, Springer, New York, NY, USA, 1995.

[2] M. Pašić, "Fractal oscillations for a class of second order linear differential equations of Euler type," Journal of Mathematical Analysis and Applications, vol. 341, no. 1, pp. 211-223, 2008.

[3] M. K. Kwong, M. Pašić, and J. S. W. Wong, "Rectifiable oscillations in second-order linear differential equations," Journal of Differential Equations, vol. 245, no. 8, pp. 2333-2351, 2008.

[4] M. Pašić and J. S. W. Wong, "Rectifiable oscillations in secondorder half-linear differential equations," Annali di Matematica Pura ed Applicata: Series 4, vol. 188, no. 3, pp. 517-541, 2009.

[5] M. Pašić and S. Tanaka, "Fractal oscillations of self-adjoint and damped linear differential equations of second-order," Applied Mathematics and Computation, vol. 218, no. 5, pp. 2281-2293, 2011.

[6] Y. Naito, M. Pašić, S. Tanaka, and D. Žubrinić, "Fractal oscillations near domain boundary of radially symmetric solutions of $p$-Laplace equations, fractal geometry and dynamical systems in pure and applied mathematics," Contemporary Mathematics American Mathematical Society. In press.

[7] W. A. Coppel, Stability and Asymptotic Behavior of Differential Equations, D. C. Heath and Company, Boston, Mass, USA, 1965.

[8] P. Hartman, Ordinary Differential Equations, Birkhäuser, Boston, Mass, USA, 2nd edition, 1982.

[9] F. Neuman, "A general construction of linear differential equations with solutions of prescribed properties," Applied Mathematics Letters, vol. 17, no. 1, pp. 71-76, 2004.

[10] F. Neuman, "Structure of solution spaces via transformation," Applied Mathematics Letters, vol. 21, no. 5, pp. 529-533, 2008.

[11] P. Borgnat and P. Flandrin, "On the chirp decomposition of Weierstrass-Mandelbrot functions, and their time-frequency interpretation," Applied and Computational Harmonic Analysis, vol. 15, no. 2, pp. 134-146, 2003.

[12] E. J. Candès, P. R. Charlton, and H. Helgason, "Detecting highly oscillatory signals by chirplet path pursuit," Applied and Computational Harmonic Analysis, vol. 24, no. 1, pp. 14-40, 2008.

[13] S. Jaffard and Y. Meyer, "Wavelet methods for pointwise regularity and local oscillations of functions," Memoirs of the American Mathematical Society, vol. 123, no. 587, pp. 1-110, 1996.

[14] Y. Meyer and H. Xu, "Wavelet analysis and chirps," Applied and Computational Harmonic Analysis, vol. 4, no. 4, pp. 366-379, 1997.

[15] G. Ren, Q. Chen, P. Cerejeiras, and U. Kaehle, "Chirp transforms and chirp series," Journal of Mathematical Analysis and Applications, vol. 373, no. 2, pp. 356-369, 2011.

[16] M. Képesi and L. Weruaga, "Adaptive chirp-based timefrequency analysis of speech signals," Speech Communication, vol. 48, no. 5, pp. 474-492, 2006.

[17] L. Weruaga and M. Képesi, "The fan-chirp transform for nonstationary harmonic signals," Signal Processing, vol. 87, no. 6, pp. 1504-1522, 2007.

[18] E. Barlow, A. J. Mulholland, A. Nordon, and A. Gachagan, "Theoretical analysis of chirp excitation of contrast agents," Physics Procedia, vol. 3, no. 1, pp. 743-747, 2009. 
[19] M. H. Pedersen, T. X. Misaridis, and J. A. Jensen, "Clinical evaluation of chirp-coded excitation in medical ultrasound," Ultrasound in Medicine and Biology, vol. 29, no. 6, pp. 895-905, 2003.

[20] T. Paavle, M. Min, and T. Parve, "Using of chirp excitation for bioimpedance estimation: theoretical aspects and modeling," in Proceedings of the 11th International Biennial Baltic Electronics Conference (BEC'08), pp. 325-328, Tallinn, Estonia, October 2008.

[21] M. Pašić, "Rectifiable and unrectifiable oscillations for a class of second-order linear differential equations of Euler type," Journal of Mathematical Analysis and Applications, vol. 335, no. 1, pp. 724-738, 2007.

[22] K. Falconer, Fractal Geometry: Mathematical Foundations and Applications, John Wiley \& Sons, Hoboken, NJ, USA, 1999.

[23] P. Mattila, Geometry of Sets and Measures in Euclidean Spaces: Fractals and Rectifiability, vol. 44 of Cambridge Studies in Advanced Mathematics, Cambridge University Press, Cambridge, UK, 1995.

[24] K. J. Falconer, "On the Minkowski measurability of fractals," Proceedings of the American Mathematical Society, vol. 123, no. 4, pp. 1115-1124, 1995.

[25] C. Q. He and M. L. Lapidus, Generalized Minkowski Content, Spectrum of Fractal Drums, Fractal Strings and the Riemann Zeta-Function, vol. 127 of Memoirs of the American Mathematical Society, American Mathematical Society, Providence, RI, USA, 1997.

[26] M. L. Lapidus and M. van Frankenhuijsen, Fractal geometry, Complex Dimensions and Zeta Functions: Geometry and Spectra of Fractal Strings, Springer Monographs in Mathematics, Springer, New York, NY, USA, 2006.

[27] M. Pašić, "Minkowski-Bouligand dimension of solutions of the one-dimensional p-Laplacian," Journal of Differential Equations, vol. 190, no. 1, pp. 268-305, 2003.

[28] J. S. W. Wong, "On rectifiable oscillation of Euler type second order linear differential equations," Electronic Journal of Qualitative Theory of Differential Equations, vol. 2007, no. 20, pp. 1-12, 2007.

[29] M. Pašić, "Rectifiable and unrectifiable oscillations for a generalization of the Riemann-Weber version of Euler differential equation," Georgian Mathematical Journal, vol. 15, no. 4, pp. 759-774, 2008.

[30] M. Pašić and S. Tanaka, "Rectifiable oscillations of self-adjoint and damped linear differential equations of second-order," Journal of Mathematical Analysis and Applications, vol. 381, no. 1, pp. 27-42, 2011.

[31] M. Pašić, D. Žubrinić, and V. Županović, "Oscillatory and phase dimensions of solutions of some second-order differential equations," Bulletin des Sciences Mathématiques, vol. 133, no. 8, pp. 859-874, 2009.

[32] L. Korkut and M. Resman, "Fractal oscillations of chirp-like functions," Georgian Mathematical Journal, vol. 19, no. 4, pp. 705-720, 2012. 


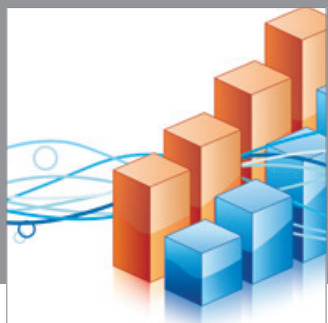

Advances in

Operations Research

mansans

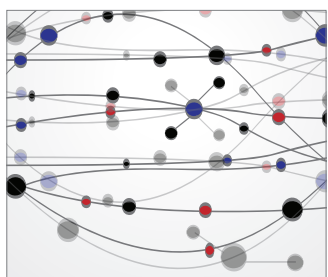

The Scientific World Journal
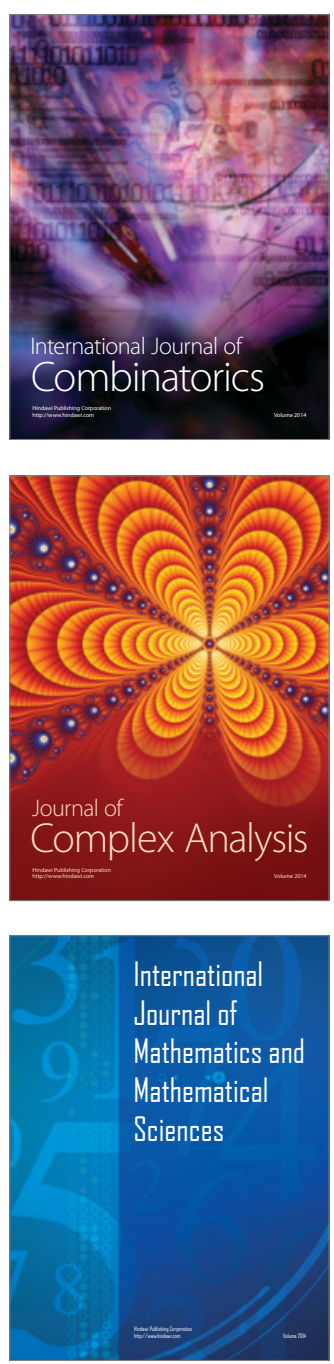
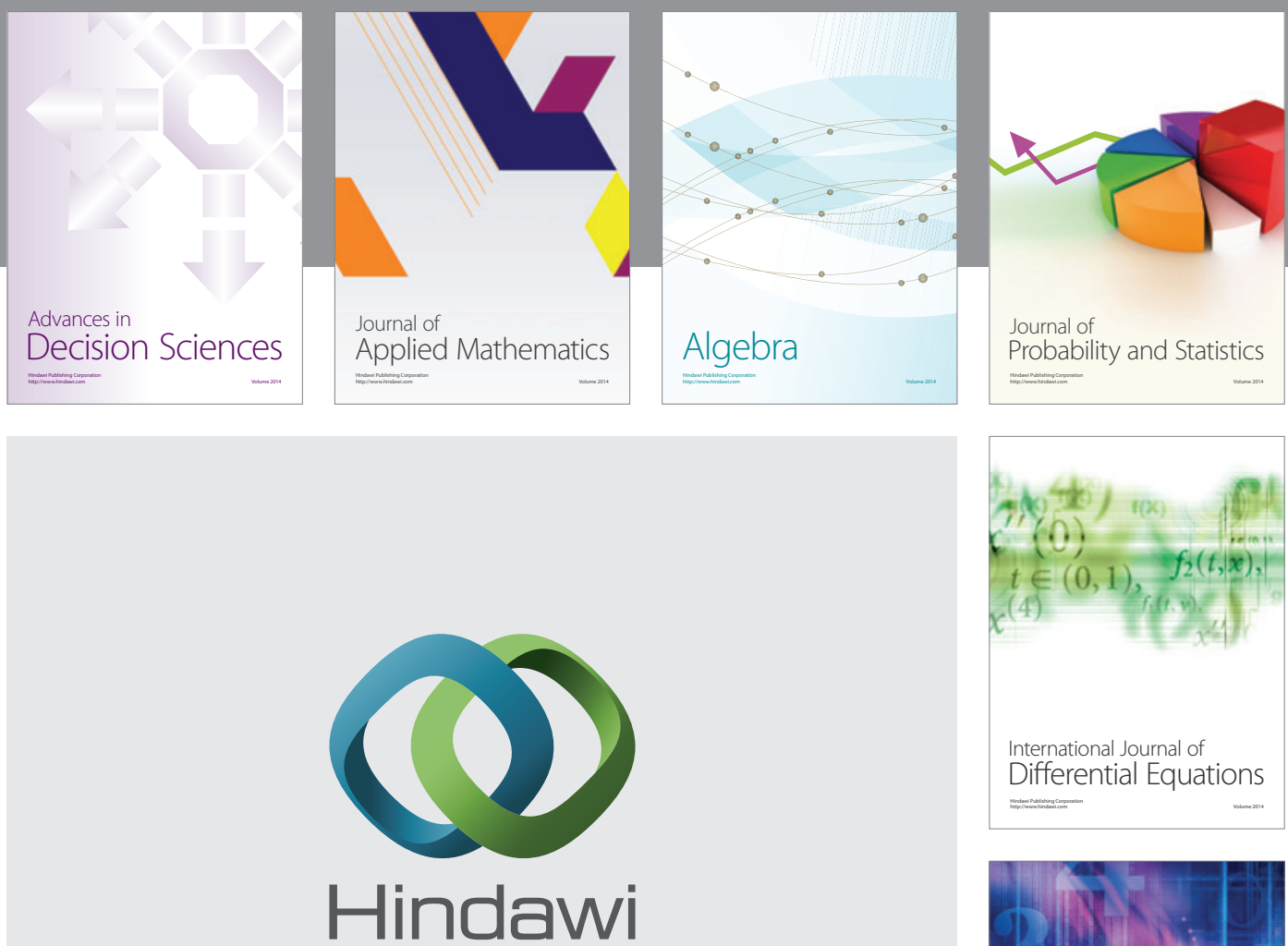

Submit your manuscripts at http://www.hindawi.com
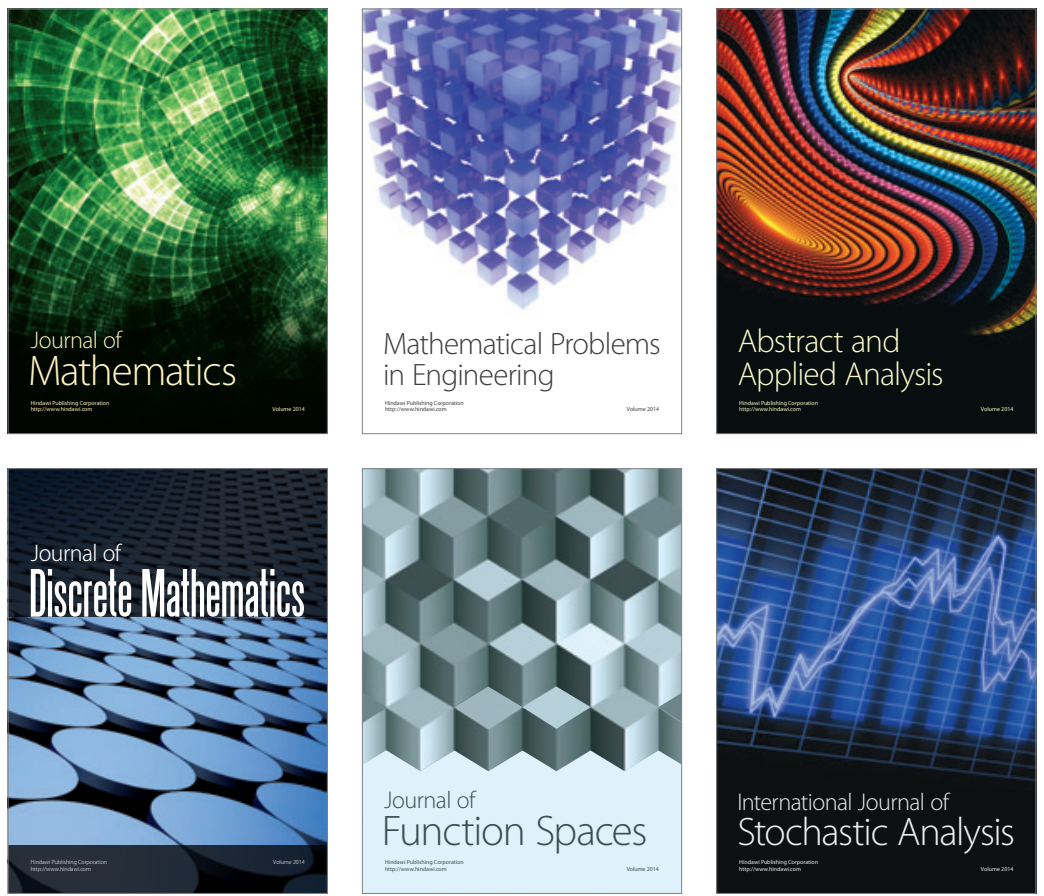

Journal of

Function Spaces

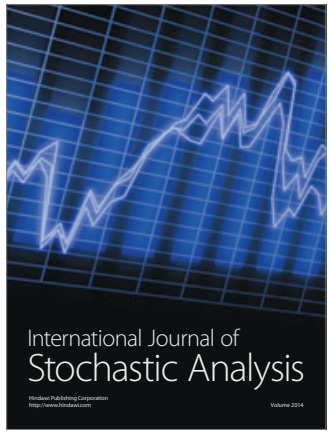

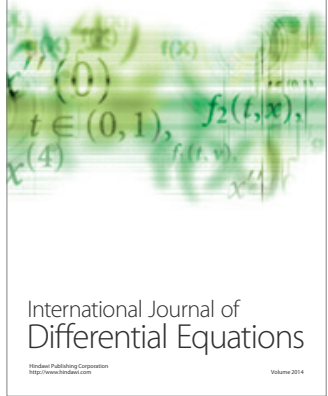
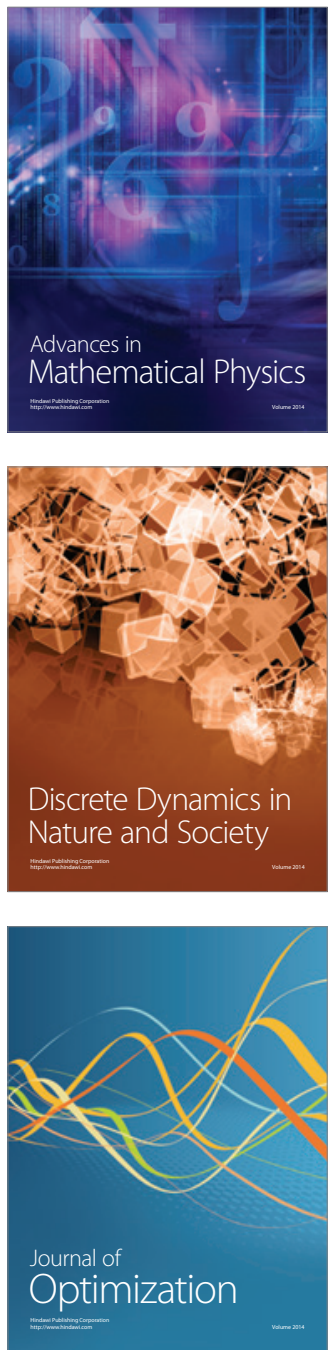